\title{
Economía afectiva
}

\section{Fabián Andrés Correa González}

y prosumidores: elementos para el abordaje de la cocreación en las industrias creativas colombianas

\section{Affective economy and prosumers: approaching co-creation in the Colombian creative industries}

http://dx.doi.org/10.18566/comunica.n43.a05

Fecha de recepción: 19 de octubre de 2020

Fecha de aceptación: 13 de noviembre de 2020

\section{Resumen}

Las industrias creativas atraviesan por importantes cambios debido a constantes desarrollos tecnológicos y a la creación de proyectos con complejas estructuras de producción que han propiciado el surgimiento del prosumidor. Este renovado actor de la producción cultural se ha vinculado a distintas iniciativas de creación aportando recursos económicos o conocimiento. Las iniciativas de creación colaborativa toman el nombre de cocreación y generan el replanteamiento de diversas facetas de la producción. Para indagar sobre este fenómeno se han revisado los postulados teóricos desarrollados en la última década acerca de la cocreación, el transmedia y el prosumidor. En el rastreo se identificó la falta de una definición clara de cocreación, la cual se debe a que los investigadores han abordado el tema de forma fragmentada, lo que imposibilita una visión completa del fenómeno. En el estudio se propone una definición concreta de cocreación, que integra a todos los elementos que la condicionan. Asimismo, se señala un camino metodológico para el desarrollo de este tipo de prácticas en las industrias creativas colombianas.

\section{Abstract}

The creative industries are going through important changes thanks the constant technological developments and the creation of projects with comunicación

número 43

Julio - diciembre

2020 | pp. $70-88$

Comunicador en lenguajes audiovisuales, Universidad de Medellín. Magíster en Comunicaciones, Universidad de Antioquia. Docente de cátedra e investigador de la Universidad de Antioquia. fabian.correa@udea.edu.co https: / / orcid.org/00000002-6778-2583

Palabras clave Cocreación, productores, prosumidor, industrias creativas, transmedia

\section{Key words}

Co-creation, producers, prosumer, creative industries, transmedia storytelling. 
complex production structures that have led to the emergence of the prosumer. This new figure of cultural production has been linked to different initiatives, providing economic resources or knowledge. These initiatives of collaborative creation have taken the name of co-creations and have modified various aspects of today's creative production. To study this phenomenon, we reviewed the theoretical postulates developed in the last decade about co-creation, transmedia and the prosumer. In our review, we identified the lack of a clear definition of co-creation, this is do to the fact that researchers have addressed the subject in a fragmented way, making it difficult to have a complete view of the phenomenon. Our study proposes a concrete definition of co-creation, which integrates all the different elements that condition it. Likewise, we point out a methodological path for the development of this type of practices in the Colombian creative industries.

\section{Introducción}

La participación de los prosumidores en el desarrollo de proyectos audiovisuales juega un papel cada vez más importante, pues en los últimos años estos actores se han transformado en un eje que dinamiza aspectos de creación y producción en las industrias creativas. La masificación de internet, el constante desarrollo de dispositivos electrónicos portables (cada vez con mayor calidad técnica y de conectividad) y la consolidación de plataformas para la creación y difusión de contenidos son algunos de los elementos que han posicionado la figura del prosumidor (Islas, 2008a, 2010). Las acciones de este renovado actor comunicativo han propiciado la revaluación de procesos como los de monetización y difusión de productos audiovisuales, la consolidación de normas de derechos de autor y propiedad intelectual, al igual que la expansión de las formas de narración, que han sido agrupadas bajo el concepto transmedia (Carrero y Pulido, 2012; García-Galera, 2014).

La definición de prosumidor se ha desarrollado durante las últimas décadas partiendo de los trabajos de McLuhan. El prosumidor se caracteriza por ser un coparticipante y cocreador de conocimientos y soluciones, como lo señalan Aguilera et al. (2014). La formación profesional del prosumidor ha sido abordada por Orihuela (2002), quien plantea que aquella no es necesaria para ser parte del contexto de las industrias creativas. Para este autor, el prosumidor participa y crea sin abandonar su lugar de receptor. Las consecuencias del accionar del prosumidor han sido abordadas tanto por Orihuela (2002) como por Gómez y Pérez (2010), quienes plantean que sus acciones han llevado a un replanteamiento de conceptos como el de derechos de autor. 
Son varias las posibles funciones de los prosumidores. Hernández Pérez y Grandío Pérez (2011) consideran que esta figura puede asumir tres funciones: difusión, interpretación y creación. Por su parte, Hayes (2015) propone cinco funciones a manera de perfiles de participación en la web 2.0: consumidor, distribuidor, crítico, editor y creador. Guerrero (2014) establece cuatro modelos de participación: observativo, argumentativo-discursivo, creativo-divulgativo y lúdico-jugador. Cabe destacar que estos estudios coinciden en que las audiencias han comenzado a ser parte de la producción cultural actual.

La calidad y volumen de aportes que un prosumidor puede ofrecer a un proceso creativo han llevado a que múltiples proyectos audiovisuales sean realizados bajo una modalidad denominada cocreación. Dentro de los variados tipos de vinculación que pueden establecer los prosumidores con un proyecto creativo, la cocreación aparece como una forma de relación y trabajo horizontal, en la que se reconoce el potencial de cada persona para aportar ideas y recursos, tomar decisiones y guiar el proyecto, todo esto con el propósito de lograr un objetivo común. El reconocimiento y nivel de implicación que logran tener los prosumidores en las cocreaciones se diferencian de los que se dan en las formas tradicionales de participación, en las cuales las personas no logran involucrarse totalmente con el proceso, pues las funciones y responsabilidades que adquieren están claramente establecidas por alguien más que limita su vinculación (Quero y Ventura, 2014; Zwass, 2010). En la cocreación, el diseño del proyecto y la forma de vinculación a este son procesos que se construyen en conjunto entre los cocreantes (Zurbriggen y Lago, 2014).

En los últimos años se han desarrollado múltiples investigaciones que buscan esclarecer las transformaciones en las industrias creativas (Bustamante, 2009) y las nuevas formas de participación (Masip y Suau, 2014). Algunas investigaciones se han enfocado en identificar las etapas por las que atraviesa un proyecto creativo (Johnson y Johnson, 1999; Quero y Ventura, 2014), mientras otras en los procesos organizativos y comunicacionales que permiten la toma de decisiones (Font y Font, 2007); otro grupo de investigaciones ha analizado el contenido de los proyectos participativos, especialmente las cualidades narrativas y estéticas como elementos que conforman comunidades de prosumidores (Ryan, 2004, 2009).

Un cuarto grupo de investigaciones ha indagado sobre la importancia de los entornos digitales para el desarrollo de proyectos colaborativos (Legorburu, 2013). Todos estos estudios nos permiten evidenciar que el abordaje de los cambios en las industrias creativas se ha hecho de forma fragmentada, puesto que no se presentan propuestas interdisciplinarias alrededor de estos fenómenos. 
Los anteriores estudios han planteado distintos conceptos para describir las relaciones de consumo, y se quedan cortos en términos de construcción conjunta de procesos. Algunos conceptos como crowdfunding1, crowdsourcing ${ }^{2}$ y precompra se han usado para especificar formas de participación o actividades de los prosumidores. En este contexto, la cocreación aparece como un tipo de vinculación ideal. Y aunque esta forma de creación se ha convertido en una gran apuesta de trabajo para múltiples proyectos de las industrias creativas, todavía no son claros los límites que la separan de otras formas de creación.

Con el fin de analizar la cocreación como una nueva forma de desarrollar procesos creativos hemos rastreado las principales propuestas teóricas sobre el fenómeno, para posteriormente contrastarlas con dos estudios de caso y así evidenciar los alcances y límites de la cocreación. Los postulados teóricos se han centrado en identificar los factores contextuales y condicionantes de las formas de participación, como la apropiación de los prosumidores de los entornos digitales; en los estudios de caso, estos factores deben ser controlados por aspectos legales y económicos.

En los estudios de caso identificamos que se han centrado en afianzar metod ologías de participación en las que son utilizados los entornos digitales para la captación y procesamiento de los aportes de los prosumidores. Lo anterior nos permite plantear que para esclarecer la cocreación como fenómeno se deben retomar propuestas teóricas y experiencias prácticas con el fin de abordar todas las variables que intervienen sobre esta forma de creación, para así hacer propuestas metodológicas que apunten al abordaje y desarrollo de estos procesos colaborativos. La ejecución de una cocreación, en este contexto, se convierte en una ocasión para el intercambio de saberes y recursos de un grupo de prosumidores y creadores iniciales con una meta en común, la de plantear una nueva economía afectiva.

\section{Hacia una definición de cocreación}

Aunque en el contexto de las industrias creativas las formas de creación colaborativa se han vuelto frecuentes, todavía existe un vacío en la definición de cocreación, una definición que explique sus características y sus diferencias con la participación. Con el propósito de solucionar este vacío teórico nos propusimos construir una definición partiendo de estudios sobre transmedia y prosumidor, puesto que en estos también son abordados los usuarios activos y las relaciones que estos establecen con los medios y con otros prosumidores alrededor del contenido y de los relatos que integran un proyecto (Meso et al., 2014; Thomas y Dyson, 2007).
Forma de vinculación a los proyectos creativos en la que los prosumidores realizan aportes económicos con el propósito de que alcance el objetivo establecido. Se diferencia de la inversión en que no hay un compromiso directo de retribución de los aportes (se aproxima a la figura de mecenazgo).

2 Forma de vinculación a los proyectos creativos en la que los prosumidores aportan ofreciendo asesoría especializada, es decir, ponen a disposición de un proyecto su conocimiento especializado, con el propósito de que alcance el objetivo establecido. 
En los estudios de transmedia y prosumidor se han examinado las características de las relaciones prosumidor-contenido, prosumidorproductor y prosumidor-prosumidor. Asimismo, se han indagado otras variables como la forma en que los entornos digitales, las legislaciones y los géneros audiovisuales determinan las posibilidades de expansión de un universo narrativo o las de participación de las comunidades (Castells, 2009; Costa, 2013; Fernández, 2014; Islas, 2008b; Jenkins, 2003).

Algunas disciplinas que han abordado la cocreación son el mercadeo (García Rodríguez et al., 2011), la administración (Mutis y Ricart, 2008) y la educación (Aparici y Silva, 2012). Dentro del contexto de las industrias creativas, también se han desarrollado estudios en el tema. John Banks, investigador de las industrias creativas, en entrevista con Jenkins (2014) plantea que la cocreación es la práctica en la que los prosumidores tienen un papel activo en la generación de valor para la producción y el consumo cultural.

Desde el campo de la administración se concibe a la cocreación como una relación entre empresas y prosumidores que contribuye a valorizar el producto y el mercado. Zwass (2010) fundamenta sus investigaciones sobre la cocreación en la visión reduccionista del consumidor, asumido como un comprador pasivo que responde a los cambios, pero no como quien los genera. Esta concepción no ha permitido entrever los roles que los prosumidores pueden asumir y que afectan al mercado, muchos de ellos motivados por aspectos que superan lo monetario y alcanzan el plano de las emociones. Las cocreaciones no deben ser entendidas, entonces, como la personalización de los productos, sino como la creación de bienes a través de nuevas formas y medios de desarrollo.

Uno de los aspectos de la cocreación más analizados desde diferentes disciplinas es la interacción entre prosumidores. En estos análisis se han abordado los principios para gestionar las interacciones y desarrollar cocreaciones. Prahalad y Ramaswamy (2004) proponen la metodología DART para la creación de valor agregado en productos a partir de la relación de empresas con prosumidores. Esta metodología se sustenta en cinco elementos: el diálogo, el diagnóstico, el acceso, el riesgo y la transparencia. Las actividades en busca de solución a problemáticas que aquejan a los prosumidores han sido investigadas por Johnson y Johnson (1999), quienes, desde la pedagogía, proponen la interacción promotora como una forma de unificar esfuerzos para desarrollar tareas grupales, todo regido bajo el precepto del bien común. El creador inicial de la cocreación es quien debe gestionar y acompañar las relaciones entre prosumidores, debido a que estas se constituyen en el pilar central del proceso. 
Las discusiones académicas sobre la cocreación han abordado consistentemente la influencia de los entornos digitales. Los estudios presentan evidencias acerca de cómo las redes sociales se han transformado en espacios de encuentro y discusión entre prosumidores y empresas, posibilitando que el inicio de una cocreación sea planteado por cualquier actor de las industrias creativas. Gracias a la importancia de estos entornos, es fundamental que las relaciones entre todos los involucrados sean mediadas y gestionadas, ya sea por el creador inicial o por un tercero que se adhiera al proyecto, teniendo en cuenta las lógicas que proponen estos escenarios digitales.

Considerando los postulados teóricos referenciados, proponemos entender la cocreación como una interacción comunicativa que busca dar respuesta a un problema. En este proceso, los aportes de los participantes deben tener el mismo valor y materializarse a través de diferentes medios. La cocreación es intencionada y estimulada por un creador inicial, quien la coordina. Una vez comienza el trabajo en conjunto, no debe haber limitación en las formas de participación. Adicionalmente, la cocreación se puede dar de forma asincrónica, pues los entornos digitales posibilitan la circulación de información en cualquier momento y hacia cualquier lugar, permitiendo así que una creación conjunta sea desarrollada por prosumidores en diferentes lugares del mundo (Jenkins, 2014; Johnson y Johnson, 1999; Prahalad y Ramaswamy, 2004; Zwass, 2010).

Finalmente, la cocreación se caracteriza por reconocer como creadores a quienes pueden aportar en distintos ámbitos del proceso: diseño, financiación, desarrollo, prueba o divulgación del proyecto. Esta característica marca la principal diferencia entre participación y cocreación: en la primera, el creador inicial define claramente los roles y niveles de injerencia de los participantes, mientras que en la segunda estas determinaciones son establecidas por consenso entre los miembros, bajo la gestión del creador inicial.

\section{Propuesta de economía afectiva}

Henry Jenkins (2008), en su obra Convergence culture, define la economía afectiva como una nueva configuración de la teoría de la mercadotecnia. Para Jenkins, en la industria mediática se continúan analizando las bases emocionales de la toma de decisiones del consumidor como una fuerza motriz que impulsa las decisiones de ver y comprar. De acuerdo con esta lógica, el consumidor ideal es activo, emocionalmente comprometido y socialmente interconectado, cualidades que se evidencian en los fans y prosumidores. Si un proyecto sociocultural, como lo indica Jenkins, encaja con el "capital emocional" de sus consumidores, se puede esperar que estos 
inviertan en él, tanto capital económico como emocional. Ese compromiso inicial se puede transformar en odio cuando los creadores iniciales alteren algo que la comunidad estime fundamental para su experiencia.

La economía afectiva ofrece ventajas tanto a los creadores iniciales como a los prosumidores. A los primeros les permite comprender cómo se dan las inversiones emocionales de los prosumidores en los proyectos, para así fomentar su vinculación a las iniciativas. Por otro lado, les ofrece ventajas al evidenciar su importancia en el desarrollo de proyectos y les plantea la necesidad de ser parte de las negociaciones para explotar su potencial.

\section{Metodología}

Nuestra investigación se desarrolló en cuatro fases: a) revisión literaria, b) estudios de caso, c) revisión documental y d) triangulación de datos. Durante la primera rastreamos los estudios que abordaron la cocreación en América y España entre los años 2000 y 2016; surgieron tres categorías de análisis: a) el creador inicial de la cocreación, sus funciones y características; b) las etapas y características de la cocreación, y c) el prosumidor, sus funciones y características.

En la segunda fase desarrollamos dos estudios de caso enmarcados en una industria específica: la publicidad. Las dos cocreaciones que estudiamos fueron desarrolladas por la agencia Big Holding (ahora llamada Track), perteneciente a la agencia internacional de publicidad DDB. El primer caso corresponde a una cocreación que buscaba actualizar el programa de CRM ${ }^{3}$ de la marca de café más reconocida en Colombia. El segundo caso, liderado por la entidad bancaria más grande del país, buscaba crear un producto de ahorro para jóvenes.

Ambas fueron planteadas inicialmente por la agencia a las compañías con el fin de demostrar las potencialidades de las cocreaciones. Luego de la aprobación de cada marca, comenzó el proceso. Para la selección de estos dos casos partimos de lo propuesto por Hernández et al. (2010) en su descripción de las muestras dirigidas, teniendo como limitación que los resultados obtenidos se pueden aplicar a las muestras en sí y a muestras similares en tiempo y lugar.

Los criterios para la selección de los estudios de caso fueron estos: que la intención de cocreación se hiciera explícita desde el inicio, que fueran desarrolladas en Colombia entre los años 2000 y 2016, y que el proceso se considerara finalizado por parte del creador inicial. Siguiendo a Hernández et al. (2010), también seleccionamos las dos experiencias referenciadas por
3 CRM (customer relationship management). Programas de relacionamiento y administración de las relaciones con el público objetivo de las empresas. 
ser las más sólidas entre las identificadas en Colombia. Los estudios de caso fueron abordados con dos instrumentos: la entrevista semiestructurada y la revisión documental. Las entrevistas preguntaron por las labores de cuatro áreas de la agencia de publicidad: Área Creativa, Área de Planning, Área de Producción y Área Digital.

Para la tercera fase, la revisión documental, analizamos videos, textos e imágenes con los resultados de la cocreación. A través de estas piezas, la agencia presentó a las marcas los hallazgos de los talleres con los prosumidores. Finalmente, en la fase de triangulación de datos, y partiendo de lo propuesto por Benavides y Gómez-Restrepo (2005), procesamos la información obtenida en las tres fases previas buscando los puntos convergentes y divergentes presentes entre las fuentes de información.

\section{Tres ejes para entender la cocreación: el creador inicial, el proceso y el prosumidor}

Obtenida toda la información, en la fase de triangulación trazamos tres ejes: el creador inicial de la cocreación, la cocreación como proceso y los prosumidores. Sobre cada eje clasificamos los hallazgos encontrados en los postulados teóricos y en los estudios de caso, lo que nos permitió identificar las temáticas recurrentes entre todas las fuentes de información. Con este proceso identificamos temas que solo han sido analizados desde uno de estos ejes, como los legislativos (desde la visión de los creadores iniciales) y los modelos de monetización (desde los prosumidores). Asimismo, identificamos que entre algunas temáticas y algunas etapas de las cocreaciones se presentan conexiones importantes de dependencia; esto nos posibilitó esclarecer las sinergias presentes entre los procesos de creación colaborativa. Las temáticas recurrentes identificadas en los tres ejes son las que siguen:

\section{Características de un proyecto}

Los relatos dentro de una cocreación y los niveles de apertura de los creadores iniciales hacia las intervenciones de los prosumidores son dos elementos claves para el desarrollo de iniciativas colaborativas. Desde el aspecto narrativo, Costa (2013) propone cuatro características que deben tener los proyectos que desean vincular al prosumidor: a) alto grado de complejidad en la historia, b) creación de contenidos específicos para cada uno de los medios y plataformas del proceso transmedial, c) al menos tres storylines pertenecientes al mismo universo narrativo y d) el prosumidor debe participar de una o varias maneras. Estas variables están encaminadas a posibilitar un nivel de inmersión del prosumidor en el relato, lo que 
posteriormente puede desencadenar el fenómeno fan, de gran importancia para los proyectos de cocreación. Una experiencia transmedia multiplica las posibilidades de vinculación emocional conformando comunidades que, como lo plantean otros autores, son el punto inicial de una creación colaborativa (Lastra, 2016).

Contrastando los postulados teóricos con los estudios de caso, identificamos que en las cocreaciones colombianas se consideraron características únicas de cada proyecto. La agencia de publicidad centró su análisis en las marcas, abordando aspectos especiales como perfil del público objetivo, productos de cada compañía o la problemática por abordar. Las dos compañías optaron por un proceso de cocreación luego de que la agencia les mostrara la necesidad de desarrollar nuevas metodologías de relación con los clientes (como fueron nombrados los prosumidores por la agencia) y para dar respuesta a cambios sociales a través de nuevos productos; así lo manifestó uno de los profesionales de la agencia:

Existen muchas investigaciones y diagnósticos en las empresas. Internamente tienen varios diagnósticos de sus problemas, de por qué las cosas no funcionan, de qué deberían hacer, de qué es lo que el cliente está pidiendo. Hay demasiadas investigaciones, pero hay un problema en la ejecución: ¿cómo llevo esto a cabo para que llegue al cliente [prosumidor] como él lo quiere?; es ahí en donde la investigación se queda corta (...) la cocreación soluciona un poco esa ejecución, porque ya es el cliente [prosumidor] el que te está solucionando (...) se hace mucho más claro llevar la idea a la ejecución (entrevistado 3, Área Digital). ${ }^{4}$

Continuando con los estudios de caso, encontramos que la agencia de publicidad califica a los productos resultado de la cocreación como alineados con las necesidades de los clientes. Al momento de determinar si es pertinente desarrollar una cocreación, para la agencia es importante establecer el impacto que podrían tener los resultados. Una de las conclusiones a las que han llegado es que no es pertinente desarrollar cocreaciones cuando los resultados pueden causar alguna molestia en el prosumidor por traer cambios negativos, como un incremento de precios.

En los dos casos de publicidad analizados, identificamos que el posicionamiento de las marcas es determinante para optar por la cocreación. Los dolores, como les llaman a las experiencias negativas de los prosumidores con la marca, que estén previamente presentes en la mente son un mal antecedente para un proceso de cocreación del que se beneficiará la marca. 


\section{Etapas de una cocreación}

A partir de los planteamientos teóricos rastreados, identificamos dos etapas comunes a múltiples cocreaciones. La primera es la identificación del problema y la formulación de un objetivo para solucionarlo (Zurbriggen y Lago, 2014; Font y Font, 2007). En la segunda comienza la convocatoria. Quero y Ventura (2014) resaltan la importancia del reconocimiento social de los creadores iniciales para lograr la vinculación de los primeros prosumidores al proyecto. Para Roig et al. (2012), las convocatorias actúan como filtro de participación regulando el nivel de influencia que los prosumidores tendrán.

Adicionalmente, detectamos ocho etapas que no fueron desarrolladas de manera profunda por los investigadores: reformulación del proyecto, reconstrucción de las estrategias para alcanzar el objetivo, obtención de los primeros resultados, evaluación de resultados a partir del objetivo planteado, ajuste de la estrategia de cocreación, socialización de resultados, liberación del producto creado y apropiación por parte de la sociedad.

En nuestros dos estudios de caso notamos una característica común: la ausencia de los prosumidores en algunas etapas del proceso. Las dos cocreaciones comenzaron formulando el objetivo general entre la agencia y la marca. Posteriormente, la agencia planeó el proceso determinando las características que debían cumplir los prosumidores que se iban a convocar, las etapas del proceso, las formas de cocreación y las técnicas para recolección y procesamiento de información. Esta forma de construcción del proyecto evidenció que los casos abordados estaban más cercanos a la concepción de participación que a la de cocreación que formulamos para esta investigación.

La formulación del proyecto por parte de la agencia estuvo acompañada de un proceso denominado por ellos como "escucha digital". Alli observaron la presencia y opiniones sobre la marca en internet. La agencia lleva a cabo esta actividad bajo distintas acciones:

Tenemos herramientas de monitoreo de redes sociales, en donde comenzamos a extraer alrededor de ese tema qué se habla, cuáles son los tópicos que más se mencionan, análisis de sentimientos para saber si hay sentimientos positivos o negativos; es una exploración que complementa las ideas que tenemos (entrevistado 3, Área Digital).

Rastrearon varios temas relacionados con los objetivos de cada cocreación con el propósito de identificar tendencias en redes sociales. Para conocer algunos rasgos de los prosumidores, la etapa de escucha digital se realizó antes de los talleres presenciales. Después de la escucha digital, la agencia seleccionó 
a los prosumidores; según la agencia, el proceso de convocatoria fue uno de los más difíciles, puesto que muchos de los convocados no entendían la propuesta de trabajo de una cocreación: "[Los prosumidores] no tienen claridad de para qué es el espacio de cocreación, aunque nosotros como agencia lo manifestemos desde el inicio" (entrevistado 1, Área de Planning).

La vinculación de los prosumidores a la cocreación solo tuvo lugar durante los talleres presenciales, desarrollados en espacios planeados por la agencia. El taller tuvo los siguientes momentos:

- Manifiesto emotivo. La agencia explicó a los prosumidores la importancia de su participación en el taller.

- Explicación del proceso. Para incentivar la participación, la agencia explicó las dinámicas de trabajo, los propósitos de la cocreación, la duración del taller y el estado actual de la marca.

- Proceso de storytelling. Para conocer la relación entre la marca y sus prosumidores, se le preguntó a cada uno "¿cómo es tu día con la marca?".

- Momento de creación. Se les preguntó a los prosumidores “¿cómo solucionarían los problemas que tienen con la marca?". A partir de esta pregunta construyeron una propuesta de solución. Para esta actividad recurrieron a técnicas como la creación de collages, creación de piezas gráficas, diseño de productos y procesos, y diseño de textos y mensajes. La agencia dispuso material análogo como imágenes impresas, papeles y herramientas para cortar y pegar; al igual que tabletas con material audiovisual digital y programas para su edición.

- Socialización de ideas. Bajo la gestión del moderador, los prosumidores socializaron sus propuestas y discutieron los aportes de cada uno.

- En el último momento del taller, y por los intereses de la agencia, se les preguntó a los prosumidores "¿cómo esperan que la marca se comunique?". Con base en las respuestas obtenidas se evaluaron la forma y los temas de los mensajes publicitarios desarrollados para la marca.

- Cierre del taller. Se les agradeció a los prosumidores por su participación con la entrega de regalos a nombre de las marcas; estos no correspondían a productos o servicios ofrecidos por ellas.

Durante los talleres, los profesionales de la agencia gestionaron el trabajo en las mesas, con el fin de motivar y garantizar la participación de todos los prosumidores. Recolectaron sistemáticamente la información y este registro se consolidó en soportes de texto, audio y video. En ninguno de los estudios de caso fueron socializados los resultados finales con los prosumidores. Al respecto, un entrevistado indicó: "Eso está limitado por lo que la marca quiere hacer, pero sí debería haber un contacto posterior con las personas" (entrevistado 1, Área de Planning). En las propuestas teóricas, esta acción es considerada clave para que los cocreantes se sientan vinculados, debido a 
que potencializa el sentido de pertenencia por el proyecto y la comunidad, al igual que contribuye a la expansión de los resultados.

\section{Momentos de participación}

Por razones sociales, narrativas y tecnológicas, los prosumidores cuentan con distintos momentos para vincularse a las cocreaciones. Retomando la propuesta de clasificación de estas planteada por Quero y Ventura (2014) y Bellefamme et al. (2012), podemos establecer tres momentos en que los prosumidores pueden aportar a un proyecto: el objetivo por alcanzar en cada fase, la etapa de desarrollo en la que esté el proyecto y el estado de la relación entre prosumidores y creadores iniciales. A partir de estos momentos, Quero y Ventura (2014) plantean el colanzamiento, la cogeneración de ideas, el codiseño, la coevaluación, el cotest, la cofinanciación y el coconsumo como maneras de contribución y etapas.

Para que los prosumidores se vinculen a las distintas fases, es necesario evaluar el nivel de apertura de los creadores iniciales. Entonces, hay que analizar dos aspectos: el nivel de implicación, en términos afectivos y materiales, de los prosumidores y el nivel de apertura que los creadores iniciales tengan hacia las intervenciones de aquellos. Sumado a lo anterior, está la descentralización controlada de una parte de la actividad creativa; para Roig (2010), esta característica se traduce en la construcción de reglas de juego claras.

Es necesario ahora clarificar la diferencia entre participación y cocreación. Para esto, es pertinente revisar la propuesta de Pateman (1970), quien afirma que en una participación parcial las partes se influyen mutuamente en la toma de decisiones, pero la decisión final es tomada por una de ellas. Por otro lado, la participación total se define como el proceso en el que las partes tienen el mismo poder para tomar decisiones, lo que en nuestro caso asumimos como cocreación. Esta propuesta muestra cómo la noción de participación se vincula a otros conceptos como poder o control, importantes para establecer fronteras entre participación y cocreación.

Analizando la forma en la que los prosumidores pudieron aportar a las cocreaciones que estudiamos en Colombia, evidenciamos que en ambos casos se ciñeron a las directrices establecidas por la agencia. En términos materiales, los aportes de los prosumidores giraron alrededor de la creación de collages, textos o propuestas visuales en los que mostraban sus ideales de las marcas. La agencia obtuvo muchos más aportes en el encuentro presencial: estos le permitieron registrar opiniones, actitudes y gestos que se convirtieron en aportes adicionales para el proceso, aunque algunos no fueran intencionados o conscientes: "Todo lo que digan es valioso: la 
edad, dónde vive, qué estudió, qué quiere; en todo lo que uno obtenga de sus respuestas puede encontrar similitudes con los demás" (entrevistado 5, Área de Producción). Estos aportes fueron considerados por la agencia como el material más importante para construir las propuestas

\section{Herramientas para la cocreación: importancia de lo digital}

Por ser considerados determinantes en la interacción con el prosumidor, los entornos digitales fueron los más referenciados en la revisión de literatura y en los estudios de caso. Fernández (2014) afirma que, en el actual contexto de las comunicaciones digitales móviles, se estimulan la creatividad y la autonomía de los prosumidores, logrando así que la colaboración se convierta en una práctica distintiva. En un análisis sobre el impacto de las redes sociales en las cocreaciones, Piller et al. (2011) dicen que aquellas son el medio más inmediato de relación con prosumidores.

La agencia de publicidad reconoció que internet da mayores posibilidades a los procesos de cocreación, ya que permite vincular mayor cantidad de prosumidores de diferentes lugares:

El internet es la mejor herramienta, en un punto podemos volcar el ejercicio a un tiempo real a través de Facebook Live, o a través de Twitter. Las posibilidades son infinitas en las redes, podemos llegar a personas que viven en cualquier lugar, en cualquier momento, sin fronteras (entrevistado 4, Área Creativa).

Dentro de las posibilidades que ofrece internet, también se mencionó un mayor grado de sinceridad de los prosumidores en sus respuestas, gracias al anonimato, y el hecho de poder establecer comunicación con aquellos en un entorno con códigos establecidos.

A pesar de lo anterior, la agencia considera que para conocer a los prosumidores no es suficiente la información que se obtiene de su interacción en la red. Las posturas de los prosumidores en redes sociales pueden estar condicionadas por los primeros pronunciamientos de algún prosumidor, lo que trae como resultado espacios regidos por la opinión pública donde son limitadas las posibilidades de conocer los pensamientos reales de cada persona, y esto afecta el grado de confiabilidad en las respuestas obtenidas.

\section{Discusión final}

Con el propósito de desarrollar metodologías que contribuyan a la efectiva vinculación de prosumidores a procesos creativos, proponemos cuatro elementos sobre los cuales las investigaciones académicas y las industrias 
creativas deben continuar indagando. Estos elementos se relacionan con el entendimiento de las nuevas funciones que deben desarrollar los actores involucrados en las cocreaciones, diferenciar todas las formas de participación, comprender la importancia de conocer los elementos contextuales que influyen en el desarrollo de estas formas de creación en Colombia y seguir ahondando en la conceptualización de la economía afectiva.

\section{Cambios en el papel de creador inicial}

A las actividades de gestión de recursos y financiación de proyectos por parte de los creadores iniciales, se suma ahora la gestión de relaciones entre los actores involucrados en la creación. El creador inicial debe diseñar un sistema de recompensas para retribuir los aportes de los prosumidores que, como ya se sabe, superan la esfera de lo económico y alcanzan la emocional. En las cocreaciones, las recompensas monetarias comienzan a perder relevancia respecto al reconocimiento social y la vivencia de experiencias colectivas, fundamentadas en el bienestar común.

Los prosumidores pueden vincularse en diferentes fases de la cocreación, lo que se traduce en distintas maneras de aportar. La relación entre creadores iniciales y prosumidores no solo se debe gestionar en las etapas posteriores a la creación de la obra, como sucedía en el esquema tradicional de producción: esta gestión debe comenzar desde la concepción del proyecto.

\section{Diferenciar entre participación y cocreación}

Las restricciones de participación, así como la administración y el ejercicio del poder en los procesos creativos, son determinantes para establecer si un proyecto tiene estructura de cocreación o de participación. El tipo de estructura que se establezca en el proyecto condiciona las posibilidades de apropiación del producto resultante. Estas limitaciones o libertades son determinadas por los intereses de los creadores iniciales sobre el producto por desarrollar.

Las experiencias de creación colectiva desarrolladas por la agencia de publicidad fueron denominadas por ellos cocreaciones; sin embargo, sustentados en la definición que hemos construido, no podrían ser entendidas bajo este concepto. Cuando existen intereses económicos o de propiedad, la limitación en la participación de prosumidores y la exclusión en ciertas fases nos indican que estamos realmente frente a un proceso de participación. Las experiencias llevadas a cabo por la agencia se constituyen en ejemplos claros de participación, principalmente por su ejecución y el control del proceso. 
En un nuevo escenario de producción creativa se dan el trabajo y la gestión de las cocreaciones con total reconocimiento del prosumidor en la toma de decisiones. Aquellas serán posibles en equipos abiertos, enmarcados en una propuesta de trabajo concreta, que busca un propósito explícito. Esta meta común crea un compromiso y una identidad compartida, lo que posibilita que la comunidad trascienda en el tiempo y que no dependa del creador inicial para mantenerse cohesionada.

\section{Situación en Colombia y posibles cambios}

En Colombia no se han llevado a cabo aún investigaciones acerca de la cocreación que aborden formas de vinculación de prosumidores. Esta ausencia de estudios se relaciona con la falta de propuestas de cocreación nacionales. Durante la etapa de formulación de este proyecto hicimos un rastreo de cocreaciones en el cine y la televisión del país. En el primero no identificamos ninguna; en el segundo, identificamos dos casos de participación, mas no acciones de cocreación. Estos casos son las secciones "El Cazanoticias" de Noticias RCN (2016) y "El periodista soy yo" (2016) de Noticias Caracol. En el sector audiovisual colombiano aún no se desarrollan cocreaciones que vinculen al prosumidor como fuerza creativa; es la publicidad la industria que ha incursionado en esos procesos, aunque con las limitaciones y aciertos mencionados en los casos de estudio que abordamos.

El punto de partida para desarrollar cocreaciones en el sector creativo colombiano se encuentra en la adecuada gestión de los entornos digitales. A partir de nuestros hallazgos se podría dar inicio a la formulación de técnicas para ejecutar proyectos que respondan a las cualidades que los prosumidores han adquirido en estos entornos. Estas técnicas están vinculadas con las etapas del proceso y los actores involucrados. En primera medida, con la información acá presentada se podrían establecer procedimientos en los que se detallen la formulación, ejecución y registro de información de los talleres y encuentros presenciales de una cocreación. En segundo lugar, desde el uso de sistemas de información es posible determinar procedimientos sobre el uso y gestión de los entornos digitales para la consolidación de la comunidad cocreadora. Finalmente, las variables técnicas pueden aportar al plano contractual entre los actores en términos de desarrollo de acuerdos claros de comunicación, participación y propiedad sobre los proyectos.

\section{Potencialidades de la economía afectiva}

La economía afectiva permite comprender las motivaciones de participación y la forma de vinculación de los prosumidores con una cocreación. Este tipo de relación plantea un escenario donde se reconoce un sistema económico 
en el que dos actores hacen una transacción con intereses particulares y en un escenario con variables claras. En este proceso transaccional de afectos, el reconocimiento de los prosumidores es clave para las creaciones colectivas.

Es importante que los actores involucrados en este sistema económico comiencen a ser parte activa de su funcionamiento. Es necesario que los prosumidores sean conscientes de la importancia de su participación. La economía afectiva permite a los creadores iniciales explotar el poder de la inteligencia colectiva para conducir a los prosumidores hacia un fin concreto y, al mismo tiempo, permite a los prosumidores forjar sus propias estructuras de negociación colectiva.

\section{Conclusiones}

as cocreaciones deben ser entendidas como un proceso de interacción comunicativa que busca solucionar un problema con base en los aportes de los actores involucrados. Se trata de un proceso intencionado y coordinado por el creador inicial bajo directrices que se establecen por consenso. Para que un encuentro cocreativo entre los creadores iniciales y prosumidores sea óptimo, se deben gestionar las relaciones a partir de la economía afectiva. Esta economía se plantea como un sistema coordinado por un actor concreto, el creador inicial, pero creado y regulado por todos para dar respuesta a las necesidades y deseos de los integrantes. El reconocimiento que se les dé a los participantes es determinante para la vinculación de prosumidores, puesto que estos ponen a disposición de la cocreación su capital emocional y, por consiguiente, su trabajo y recursos, con el propósito de vivir una experiencia (de reconocimiento) que debe estar sustentada en un sistema de recompensas dinámico y alineado a sus deseos.

Las experiencias transmedia son un campo de exploración para entender el accionar del prosumidor. Analizar fenómenos como las apropiaciones que los prosumidores hacen de los universos narrativos y cómo estos se transforman de un punto inicial y básico de audiencia a uno final e ideal de prosumidor es necesario para establecer técnicas de vinculación de este a las cocreaciones.

Para terminar, el abordaje de la cocreación desde la economía afectiva contribuye a identificar la naturaleza de cada proyecto. Esto nos permite evaluar si realmente las iniciativas que se presentan como cocreaciones lo son, debido a que pueden estar inmersas en una confusión con otras formas de interacción entre creativos y prosumidores, como posibilidades de acceso a información, interactividad, disponibilidad de información o participación. Tener esta claridad frente a cómo se está dando la relación 
entre creadores y prosumidores permitiría la reivindicación de otros tipos de encuentro entre estos dos actores, como lo son la participación o el acceso a la información, que en la actualidad han pasado a un segundo plano por el apogeo del término cocreación, que, por cierto, no debe ser entendida solo como participativa, sino como vinculante.

\section{Referencias}

Aguilera, I., Barros, A., Contreras, E., Galza, P., Gatica, M., Inostroza, J. y Westermeyer, N. (2014). Co-creación para la innovación: un caso en el sector público chileno. Revista Ingeniería de Sistemas, 28 (1), 5-26.

Aparici, R. \& Silva, M. (2012). Pedagogía de la interactividad. Comunicar, 19 (38).

Bellefamme, P., Lambert, T. y Schwienbacher, A. (2012). Individual crowdfunding practices. Venture Capital: an international journal of entrepreneurial finance, 15 (4), 313-333.

Benavides, M. O. y Gómez-Restrepo, C. (2005). Métodos en investigación cualitativa: triangulación. Revista Colombiana de Psiquiatría, 34 (1), 118-124.

Bustamante, E. (2009). De las industrias culturales al entretenimiento. La creatividad, la innovación... Viejos y nuevos señuelos para la investigación de la cultura. Diálogos de la Comunicación, 78, 1-25.

Caracol Televisión. (2016). El periodista soy yo [Noticiero de televisión]. Noticias Caracol.

Carrero, J. S. y Pulido, P. C. (2012). De cara al prosumidor: producción y consumo empoderando a la ciudadanía 3.0. Revista ICON014, 10 (3), 62-84.

Castells, M. (2009). Comunicación y poder. Alianza.

Costa, C. (2013). Narrativas transmedia nativas: ventajas, elementos de la planificación de un proyecto audiovisual transmedia y estudio de caso. Historia y Comunicación Social, 18 (1), 561-574.

Department for Culture, Media and Sport [DCMS]. (1998). Creative industries mapping document.

Department for Culture, Media and Sport [DCMS]. (2000). Creative industries: the regional dimension, the report of the regional Issues Working Group.

Fernández, P. (2014). Consumos culturales en América Latina y la emergencia del prosumidor: un recorrido conceptual desde la sociedad de la información. Revista Communication Papers, 4 (3), 87-100.

Font, J. y Font, C. J. (2007). Ciudadanos y decisiones públicas. Ariel.

García-Galera, M. C. (2014). Prosumidores mediáticos. Cultura participativa de las audiencias y responsabilidad de los medios. Comunicar, 22 (43), 10-13.

García Rodríguez, N., Álvarez Álvarez, B. y Santos Vijande, M. (2011). Aplicación de la Lógica Dominante del Servicio (LDS) en el sector turístico: el marketing interno como antecedente de la cultura de co-creación de innovaciones con clientes y empleados. Cuadernos de Gestión, 11 (2).

Gómez, F. y Pérez, J. (2010). Paradigmas de la producción audiovisual en la web 2.0. Razón y Palabra, 15 (72), 0-15. 
Guerrero, M. (2014). Webs televisivas y sus usuarios: un lugar para la narrativa transmedia. Los casos de Águila Roja y Juego de Tronos en España. Comunicación y Sociedad Nueva Época, 21 (1), 239-267.

Hayes, G. (2015). The myth of web 2.0 Non-Participation. Personalize media [Mensaje en un blog]. http://www.personalizemedia.com/?s=editors\&searchsubmit

Hernández, R., Fernández, R. y Baptista, M. (2010). Metodología de la investigación. McGraw Hill.

Hernández Pérez, M. y Grandío Pérez, M. M. (2011). Narrativa crossmedia en el discurso televisivo de ciencia ficción. Estudio de Battlestar Galactica (2003-2010). Área Abierta, 28 (1), 1-20.

Islas, O. (2008a). La sociedad de la ubicuidad, los prosumidores y un modelo de comunicación para comprender la complejidad de las comunicaciones digitales. Razón y Palabra, 13 (65).

Islas, O. (2008b). El prosumidor. El actor comunicativo de la sociedad de la ubicuidad. Palabra Clave, 11 (1), 29-39.

Islas, O. (2010). Internet 2.0: el territorio digital de los prosumidores. Revista Estudios Culturales, 3 (5), 43-63.

Jenkins, H. (2003). Transmedia storytelling: moving characters from books to films to video games can make them stronger and more compelling. MIT Technology Review.

Jenkins, H. (2008). Convergence culture: la cultura de la convergencia de los medios de comunicación. Paidós Ibérica.

Jenkins, H. (2014). Why co-creation matters: an interview with John Banks (part one) [Mensaje en un blog]. http:// henryjenkins.org/2014/05/why-co-creation-matters-aninterview-with-john-banks-part-one.html

Johnson, D. y Johnson, R. (1999). Aprender juntos y solos. Aprendizaje cooperativo, competitivo e individualista. Aique.

Lastra, A. (2016). El poder del prosumidor. Identificación de sus necesidades y repercusión en la producción audiovisual transmedia. Revista ICONO14, 14 (1), 71-94.

Legorburu, J. M. (2013). Modelos de convergencia de medios en España. I. Digitalización, concentración y nuevos soportes. CEU Ediciones.

Masip, P. y Suau, J. (2014). Audiencias activas y modelos de participación en los medios de comunicación españoles. Hipertext.net: Anuario Académico sobre Documentación Digital y Comunicación Interactiva, (12).

Meso, K., Larrondo, A., Peña, S. y Rivero, D. (2014). Audiencias activas en el ecosistema móvil. Hipertext.net, 12.

Mutis, J. y Ricart, J. E. (2008). Innovación en modelos de negocio: la base de la pirámide como campo de experimentación. Universia Business Review, (18).

Nakamura, K. (2004). Creating a ubiquitous network society: Japan, a nation built on technology. Conferencia Inaugural de CEATEC.

Orihuela, J. L. (2002). Nuevos paradigmas de la comunicación. Chasqui, (77).

Pateman, C. (1970). Participation and democratic theory. Cambridge University Press.

Piller, F., Vossen, A. y Ihl, C. (2011). From social media to social product development: the impact of social media on co-creation of innovation. DIE Unternehmung, 66 (1),1-22. 
Prahalad, C. K. y Ramaswamy, V. (2004). El futuro de la competencia: creación conjunta de valor único con los consumidores. Ediciones Gestión 2000.

Quero, M. y Ventura, R. (2014). Análisis de las relaciones de co-creación de valor. Un estudio de caso de crowdfunding. Universia Business Review, 1 (1), 128-143.

RCN Televisión. (2016). El cazanoticias [Noticiero de televisión]. RCN Televisión.

Roig, A. (2010). La participación como bien de consumo: una aproximación conceptual a las formas de implicación de los usuarios en proyectos audiovisuales colaborativos. Anàlisi Quaderns de comunicació i cultura, 40 (1), 101-114.

Roig, A., Sánchez-Navarro, J. y Leibovitz, T. (2012). ¡Esta película la hacemos entre todos! crowdsourcing y crowdfunding como prácticas colaborativas en la producción audiovisual contemporánea. Ícono 14, 10 (1), 25-40.

Ryan, M. L. (2004). Narrative across media: the languages of storytelling. University of Nebraska Press.

Ryan, M. L. (2009). From narrative games to playable stories: toward a poetics of interactive narrative. Storyworlds: A Journal of Narrative Studies, 1 (1), 43-59.

Thomas, A. y Dyson, S. (2007). Online TV and video: beyond user-generated content. Informa Telecoms and Media.

Unesco, Institute of Statistics. (2009). 2009 Unesco Framework for Cultural Statistics.

Zurbriggen, C. y Lago, M. G. (2014). Innovación y co-creación. Nuevos desafíos para las políticas públicas. Revista de Gestión Pública, 3 (2), 329-361.

Zwass, V. (2010). Co-creation: toward a taxonomy and an integrated research perspective. International Journal of Electronic Commerce, 1 (15), 11-48. 